\title{
Is a liberal transfusion strategy better than a symptomatic strategy in patients with cardiovascular disease undergoing surgical hip fracture repair?
}

\author{
Francesco Casella $\cdot$ Simone Birocchi $\cdot$ \\ Gruppo di Autoformazione Metodologica (GrAM)
}

Received: 17 May 2012/ Accepted: 14 July 2012/Published online: 8 August 2012

(C) SIMI 2012

\section{Background}

In patients with hip fracture, anemia may result as a direct consequence of the injury or surgery. Red blood cell transfusions are commonly administered to surgical patients in response to operative hemorrhage, and to treat anemia. However, the impact of transfusions on mortality and morbidity in patients with hip fracture is still unclear. A large observational study finds no beneficial effect of transfusion on mortality regardless of the presence of preexisting cardiovascular disease [1]. Other studies suggest that anemia decreases physical performance and postoperative rehabilitation in patients with hip fracture, and red blood cell transfusions improve short-term functional outcomes [2, 3]. To date, there are few randomized clinical trials (RCT) assessing the clinical impact of different transfusion thresholds. A large study involving patients in intensive care units shows that a restrictive strategy of redcell transfusion is at least as effective as and possibly superior to a liberal transfusion strategy in critically ill patients [4]. However, there are no RCTs evaluating the safety of withholding transfusion until symptoms of anemia develop in patients with hip fracture. Furthermore, the safety of symptomatic transfusion in patients with cardiovascular disease and the impact of a lower transfusion threshold on functional recovery after surgical hip repair have not been studied.

\section{F. Casella}

U.O. Medicina 3, Dipartimento di Medicina, Ospedale L. Sacco,

Università degli Studi di Milano, Milan, Italy

S. Birocchi $(\bowtie)$

U.O. Medicina 2, Dipartimento di Medicina, Ospedale L. Sacco, Università degli Studi di Milano, Milan, Italy

e-mail: simonebirocchi@gmail.com

\section{Summary}

The Transfusion Trigger Trial for Functional Outcomes in Cardiovascular Patients Undergoing Surgical Hip Fracture Repair (FOCUS) [5] is a multicentre randomized clinical trial comparing an aggressive transfusion strategy with a more restrictive strategy in patients with cardiovascular disease or cardiovascular risk factors undergoing surgical hip repair for hip fracture.

From July 2004 to February 2009, patients who were 50 years of age or older, who had cardiovascular disease, and whose hemoglobin level was below $10 \mathrm{~g}$ per deciliter after hip fracture surgery, were enrolled at 47 clinical sites in the United States and Canada. The eligibility criteria were expanded in December 2005 to include patients with cardiovascular risk factors. A total of 2,016 patients were randomized to a liberal transfusion strategy or a restrictive transfusion strategy. Patients allocated to the liberal strategy received transfusions when their hemoglobin level fell below $10 \mathrm{~g}$ per deciliter, while patients in the restrictivestrategy group were allowed to receive transfusions if symptoms or signs of anemia developed, such as chest pain, congestive heart failure, tachycardia or hypotension and relevant bleeding, or at the discretion of their physicians if the hemoglobin level fell below $8 \mathrm{~g}$ per deciliter. The primary outcome was death or an inability to walk $10 \mathrm{ft}$ without human assistance at the 60-day follow-up as assessed by telephone interview. Secondary outcomes included in-hospital acute coronary syndrome and death for any reason, residence at 30 and 60 days after randomization, and two measures of functional status at 30 and 60 days such as lower extremity physical and instrumental activities of daily living. 1,009 patients were allocated to the restrictive transfusion strategy and $59 \%$ of them did not receive any transfusion after randomization. On the 
contrary, a median of 2 units of red blood cells was transfused in the liberal-strategy group (1,007 patients).

The rates of primary outcome were not significantly different between the liberal-strategy and the restrictivestrategy groups (35.2 vs. $34.7 \%, \mathrm{CI}, P=0.90)$. Furthermore, there was no significant difference between the two groups in terms of death on 30 and 60-day follow-up. The frequencies of in-hospital acute myocardial infarction, unstable angina and death did not significantly differ between the two groups $(4.3 \%$ in the liberal vs. $5.2 \%$ in the restrictive strategy group; $99 \% \mathrm{CI}-3.3$ to 1.6$)$.

\section{Strengths of the study}

- It addresses a relevant clinical issue. The optimal transfusion threshold for patients with cardiovascular disease is unclear.

- It is the first RCT analyzing a wide population of patients with hip fracture. Previous studies were either observational studies or conducted among critical care patients.

- Good external validity: the high-risk population of the study with a mean age of more than 81 years is representative of elderly patients undergoing surgical hip repair for whom an untreated anemia would be more harmful than in younger individuals.

- Data collectors were blinded to group allocation

\section{Weaknesses of the study}

- Eligibility criteria were modified in the course of the trial, to include lower risk patients with cardiovascular risk factors, but no history of cardiovascular disease. Furthermore, a reduction of recruitment goal to 2,000 patients was decided during the course of the trial by the safety monitoring board, but the reason was not specified.

- Performance status was assessed by phone calls instead of clinical examinations.

- The relevant percentage of protocol violations was significantly superior in the liberal group (9\%) than in the restrictive one $(5.6 \%)$. Previous studies had fewer protocol violations [6].

\section{Question marks}

- The primary outcome is a composite of death and a performance status. A clinically relevant outcome, such as mortality, perhaps is too different from a functional outcome. Would it be better to consider, along with mortality, a more similar and clinically relevant endpoint?

- The authors decided to perform a trial to demonstrate the superiority of the liberal strategy. Previous trials [4] did not show any significant difference between the two strategies, but showed a significant reduction in blood transfusions for the restrictive strategy. A relevant issue is whether this strategy in non inferior to liberal strategy in terms of clinical outcomes. We wonder if, in this clinical setting, a non-inferiority trial would be more informative.

- The definition of active bleeding is not specified.

\section{Clinical bottom line}

Even in elderly patients with cardiovascular disease or cardiovascular risk factors, there is no evidence that maintaining a hemoglobin level of more than $10 \mathrm{~g}$ per deciliter in patients undergoing surgical repair for hip fracture improves clinical outcomes such as mortality, morbidity and functional recovery, compared with a restrictive strategy. On the contrary, it significantly increases the number of blood transfusions.

Acknowledgments The study was partially funded by the National Heart, Lung, and Blood Institute.

Conflict of interest None.

\section{References}

1. Carson JL, Duff A, Berlin JA et al (1998) Perioperative blood transfusion and postoperative mortality. JAMA 279:199-205

2. Halm EA, Wang JJ, Boockvar K et al (2003) Effects of blood transfusion on clinical and functional outcomes in patients with hip fractures. Transfusion. 43:1358-1365

3. Foss NB, Kristensen MT, Kehlet H (2008) Anaemia impedes functional mobility after hip fracture surgery. Age Ageing 37(2): $173-178$

4. Hébert PC, Wells G, Blajchman MA et al (1999) A multicenter, randomized, controlled clinical trial of transfusion requirements in critical care. N Engl J Med 340:409-417

5. Carson JL, Terrin ML, Noveck H, Sanders DW, Chaitman BR, Rhoads GG, Nemo G, Dragert K, Beaupre L, Hildebrand K, Macaulay W, Lewis C, Cook DR, Dobbin G, Zakriya KJ, Apple FS, Horney RA, Magaziner J; FOCUS Investigators (2011) Liberal or restrictive transfusion in high-risk patients after hip surgery. N Engl J Med 365(26):2453-2462 [Epub 2011 Dec 14]

6. Hébert PC, Yetisir E, Martin C et al (2001) Is a low transfusion threshold safe in critically ill patients with cardiovascular diseases? Crit Care Med 29(2):227-234 